\title{
Challenges of gravitational wave detection using long-baseline cavity-assisted large momentum transfer atom interferometry
}

\author{
M. Dovale-Álvarez;, D. D. Brown, A. W. Jones, C. M. Mow-Lowry, H. Miao, A. Freise
} School of Physics and Astronomy and Institute of Gravitational Wave Astronomy,

University of Birmingham, Edgbaston, Birmingham B15 2TT, United Kingdom

\begin{abstract}
Atom interferometers employing optical cavities to enhance the beam splitter pulses promise significant advances in science and technology, notably for future gravitational wave detectors. Long cavities, on the scale of hundreds of meters, have been proposed in experiments aiming to become demonstrators for gravitational wave detection at frequencies below $1 \mathrm{~Hz}$, where laser interferometers, such as LIGO, have poor sensitivity. Our group at the Birmingham Institute of Gravitational Wave Astronomy has explored the fundamental limitations of two-mirror cavities for atomic beam splitting, and established upper bounds on the temperature of the atomic ensemble as a function of cavity length and three design parameters: the cavity $g$-factor, the bandwidth, and the optical suppression factor of the first and second order spatial modes. A lower bound to the cavity bandwidth which avoids elongation of the interaction time and maximizes power enhancement was found. An upper limit to cavity length is also found for symmetric two-mirror cavities. These key limitations impact the feasibility of long-baseline detectors, which suffer from a naturally larger bandwidth and worse optical suppression of higher order optical modes. Our findings will aid the design of current and future experiments using this technology, such as the MIGA experiment in Bordeaux. In the future we aim to fully model the effect that the imperfect optical wavefronts have on the atomic transitions.
\end{abstract}

This paper is partly based on the publication Fundamental limitations of cavity-assisted atom interferometry, published on Physical Review A on 8 November 2017.

DOI:10.1103/PhysRevA.96.053820

GRAvitational-waves Sciencetechnology Symposium - GRASS2018

1-2 March 2018

Palazzo Moroni, Padova (Italy)

* Speaker. Email: 


\section{Introduction}

The Matter wave-laser Interferometer Gravitation Antenna [W] is an experiment aimed at studying gravity at large scale, and becoming a demonstrator for future gravitational wave detection in a frequency band $(100 \mathrm{mHz}-1 \mathrm{~Hz})$ currently forbidden in the most advanced ground-based optical interferometers, such as Advanced LIGO [వ].

Cavity-enhanced atom optics will be used to simultaneously interrogate several atomic clouds launched vertically from atomic fountains situated along the cavity axis. By injecting the cavities with short pulses of certain durations, a set of Mach-Zehnder atom interferometers are formed by Bragg scattering of the atoms from pairs of photons from the counter-propagating cavity fields. Each atom interferometer's signal is linked to the phase of the cavity field. Hence, the atom interferometers become sensitive to any effect modifying the optical path length in the cavity, such as the strain of a passing gravitational wave.

Each interferometric pulse is obtained by injecting a time-modulated pulse with a smooth temporal profile into the cavity. Bragg transitions are velocity-selective and thus, in order to maximize atomic flux through the pulse sequence, the pulse durations must be kept as short as possible. MIGA will therefore operate in the quasi-Bragg regime, with pulse durations in the tenths of $\mu \mathrm{s}$ scale and a total measurement time planned at $2 T=500 \mathrm{~ms}$.

The cavity offers two important advantages over using a retro-reflected high-power laser system: First, the cavity provides resonant enhancement of the interferometric beams, so that a high intra-cavity power may be achieved using a relatively low input power. Second, the cavity offers spatial filtering of the interferometric beams, effectively cleaning the laser phase fronts and thus enhancing the sensitivity of the detector. The cavity bandwidth plays a major role in the performance of the interferometer [B]]. Power enhancement and spatial filtering are both enhanced by increasing the cavity finesse. The maximal allowed beam size increases with cavity length. Both good spatial filtering and large beam sizes are desired qualities in the interferometer. The cavity bandwidth scales inversely with the product of finesse and length. Hence, it would seem that the narrower the bandwidth is, the better. There is a limit, however, below which the photon-atom interaction time increases substantially and power enhancement worsens, negating the advantage of incorporating the cavity in the first place. With the realisation of this bandwidth limit, the task becomes a balancing act between the quality of the cavity as a spatial filter of the interferometric beams and its ability to accommodate the size of the atomic cloud as it thermally expands during the measurement.

\section{Cavity-assisted Bragg beam splitters}

In an atomic Bragg beam splitter, the atoms scatter $2 n$ photons from the laser beams and acquire a momentum difference of $2 n \hbar k$, where $k$ is the wavenumber of the field. The large momentum transfer results in a factor $n$ scaling of the sensitivity to distortions of the relative laser phase with respect to a conventional $2 \hbar k$ Raman or Bragg interferometer. The state of the atom is described as an infinite superposition of momentum Bloch waves $|n \hbar k\rangle \equiv|n\rangle$ [四]:

$$
|\psi\rangle=\sum_{n=-\infty}^{+\infty} g_{n}(t) e^{i n k z}|n\rangle
$$


with the amplitudes $g_{n}(t)$ obeying:

$$
i \dot{g}_{n}(t)=\left[\omega_{r} n^{2}+\bar{\Omega} G(t)\right] g_{n}(t)+\frac{1}{2} \bar{\Omega} G(t)\left[g_{n+2}(t)+g_{n-2}(t)\right],
$$

where $\omega_{r}$ is the recoil frequency, $\bar{\Omega}$ is the peak 2-photon Rabi frequency - proportional to the laser intensity - and $G(t)$ is the envelope function of the pulse intensity, of width $\delta t^{1}$.

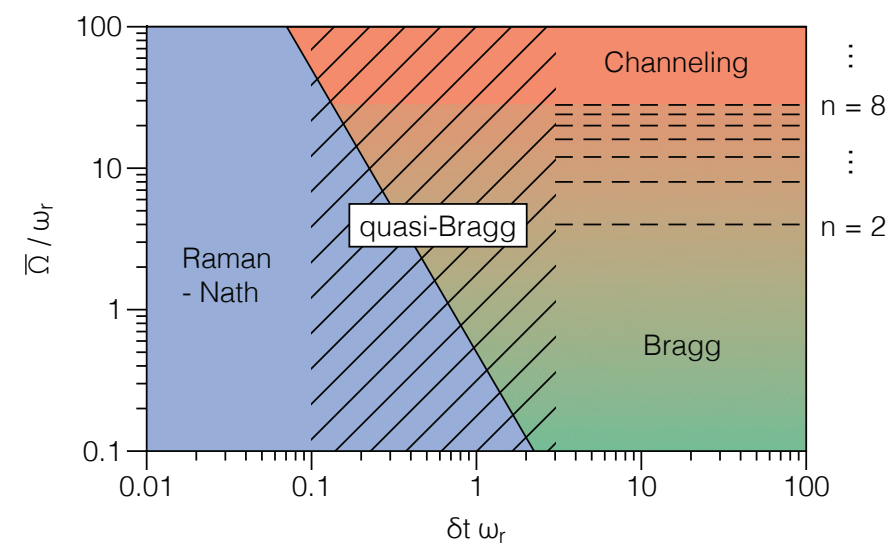

Figure 1: Parameter space of a Bragg beam splitter.

The evolution of these state amplitudes displays different behaviours on the parameter space $\delta t-\bar{\Omega}$ (depicted in Fig. 四):

- Raman-Nath regime (fast interaction): The short interaction time $\delta t \ll\left(2 \bar{\Omega} \omega_{r}\right)^{-\frac{1}{2}}$ yields a large energy uncertainty with respect to the kinetic energy term (the term $\omega_{r} n^{2}$ on the right hand side of the equation of motion). As a result, and regardless of the shape of $G(t)$, the atomic population quickly spreads over many momentum states, and the transfer efficiency to any particular state is very limited.

- Bragg regime (weak and slow interaction): In the limit of low interaction strength $\bar{\Omega} \ll$ $4(n-1) \omega_{r}$ and long interaction time $\delta t \gg 1 / \omega_{r}$, energy conservation favours transitions $|-n\rangle \rightarrow|+n\rangle$ with low losses, with the intermediate states being adiabatically eliminated, regardless of the shape of $G(t)$. The well defined energy, however, means that the pulse (with a Fourier linewidth much smaller than the recoil frequency) might be able to resolve the velocity spread of the cloud (that is typically a fraction $\frac{1}{10}$ to $\frac{1}{100}$ of the recoil velocity), hence selecting a fraction of atoms from the source.

- Channeling regime (strong and slow interaction): In this regime the shape of $G(t)$ is critical in the evolution of the atomic state. Efficient population transfer to a target state can be achieved in transitions driven at an intensity that violates the adiabacity condition, but only if the shape of $G(t)$ is smooth and the interaction time is long enough. For example, square pulses are known to give high losses of the population to the intermediate states [ 6 ].

\footnotetext{
${ }^{1}$ Throughout this paper we present all results in terms of the dimensionless interaction time or pulse width $\delta t \omega_{r}$, and the dimensionless interaction strength or intensity $\bar{\Omega} / \omega_{r}$. This makes all results readily scalable for the atomic transition of interest, with $\omega_{r}=\hbar k^{2} / 2 M$, where $M$ is the mass of the atom. For example, $\omega_{r}=23694 \mathrm{~Hz}$ for the rubidium- $87 \mathrm{D}_{2}$ transition $\left(5^{2} \mathrm{~S}_{1 / 2} \rightarrow 5^{2} \mathrm{P}_{3 / 2}\right)$, and $\omega_{r}=12983 \mathrm{~Hz}$ for the cesium- $133 \mathrm{D}_{2}$ transition $\left(6^{2} \mathrm{~S}_{1 / 2} \rightarrow 6^{2} \mathrm{P}_{3 / 2}\right)$.
} 
- Quasi-Bragg regime: This is the transition region between the short interaction RamanNath regime and the long interaction Bragg and channeling regimes [[]]. Low loss $|-n\rangle \rightarrow$ $|+n\rangle$ transitions can be achieved at short interaction times and interaction strengths which violate the adiabacity condition. The shape of $G(t)$ plays a major role in the outcome of the interaction, with smooth shapes yielding less losses.
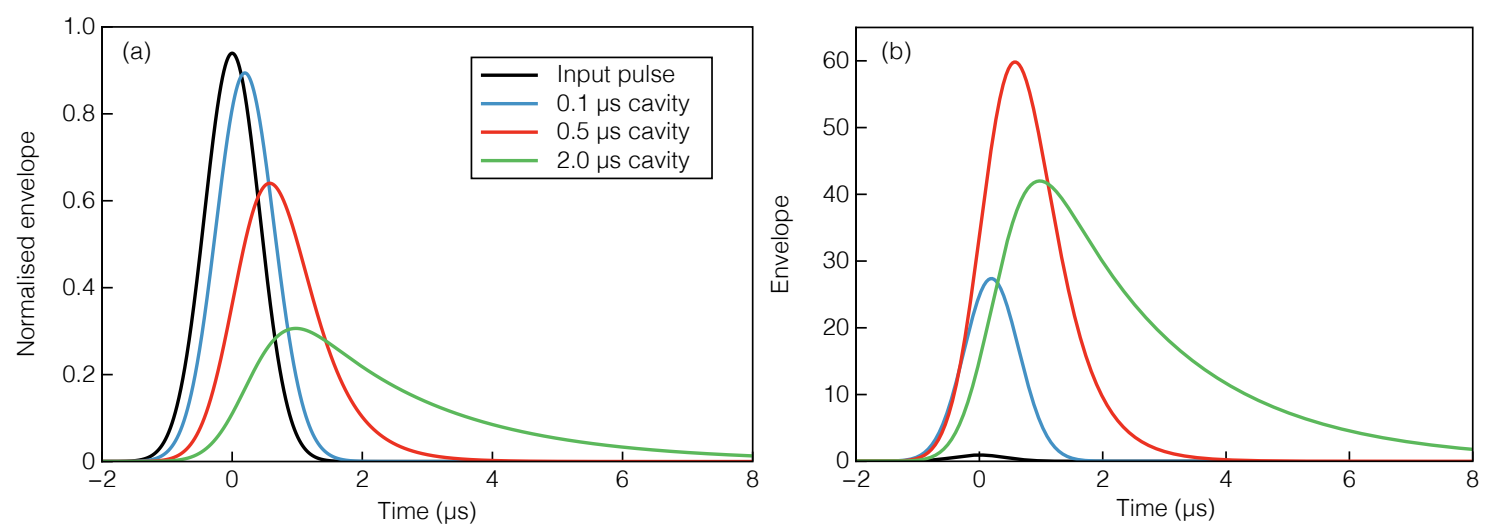

Figure 2: Dynamic response of cavities with different photon lifetimes to a $1 \mu$ s pulse. The envelope functions of the intracavity field intensity (coloured curves) are shown normalised for comparison (a) as well as non-normalised (b).

The cavity enters the problem by shaping the envelope function of the pulse $G(t) \rightarrow G^{\prime}(t)$ according to the cavity parameters. This shaping will result, ideally, in an enhanced effective interaction strength due to the increase in pulse area with respect to the input pulse, owing to the cavity's optical gain. The shape of $G^{\prime}(t)$ is univocally determined by the product of the cavity finesse $\mathscr{F}$ and length $L$. If the duration of the input pulse is of the order of the photon lifetime of the cavity $\left(\tau_{c}=\mathscr{F} L /(\pi c)\right.$ for an impedance matched and lossless cavity) or lower, the deformation of $G(t)$ will be substantial (Fig. ㅁ). This deformation is projected on the transition probabilities by shifting both the required laser intensity and the photon-atom interaction time of the beam splitter pulses, hence modifying the landscape of the parameter space depicted in Fig. $\mathrm{W}$.

By modifying the interaction time and strength, the cavity has the effect of parametrically pushing the photon-atom interactions towards the Bragg and channeling regimes. A high finesse or long baseline cavity will transform a short input pulse with a large energy uncertainty into a long pulse with a well-defined energy. In doing so, the atomic transitions with the circulating field become more adiabatic and energy conservation will favour transitions to the target state with low losses, thus operating in the slow interaction weak potential Bragg regime, unless the price is paid in terms of input power to drive transitions that violate the adiabacity condition, thus operating in the slow interaction and strong potential channeling regime. Pulses that are not short in comparison to the photon lifetime of the cavity will in turn be resonantly enhanced beyond the adiabatic limit, thus also operating in the channeling regime. In some cases, the cavity may eliminate altogether the possibility of operating in the fast interaction Raman-Nath and quasi-Bragg regimes.

By numerically integrating the equations of motion with the envelope functions shaped by the cavity, we have determined that there is an upper limit to $\tau_{c}$ (or equivalently a lower limit of the 
cavity bandwidth $\left.\Delta \omega_{c}=1 /\left(2 \pi \tau_{c}\right)\right)$ above which power enhancement of the beam splitters worsens and the minimum interaction time to achieve efficient population transfer to the target state starts increasing linearly with finesse or length (Fig. B]). This limit is dependent on the recoil frequency of the transition and on the order of the diffraction process, and is listed in Table 1 for Bragg orders $n=1-9$.
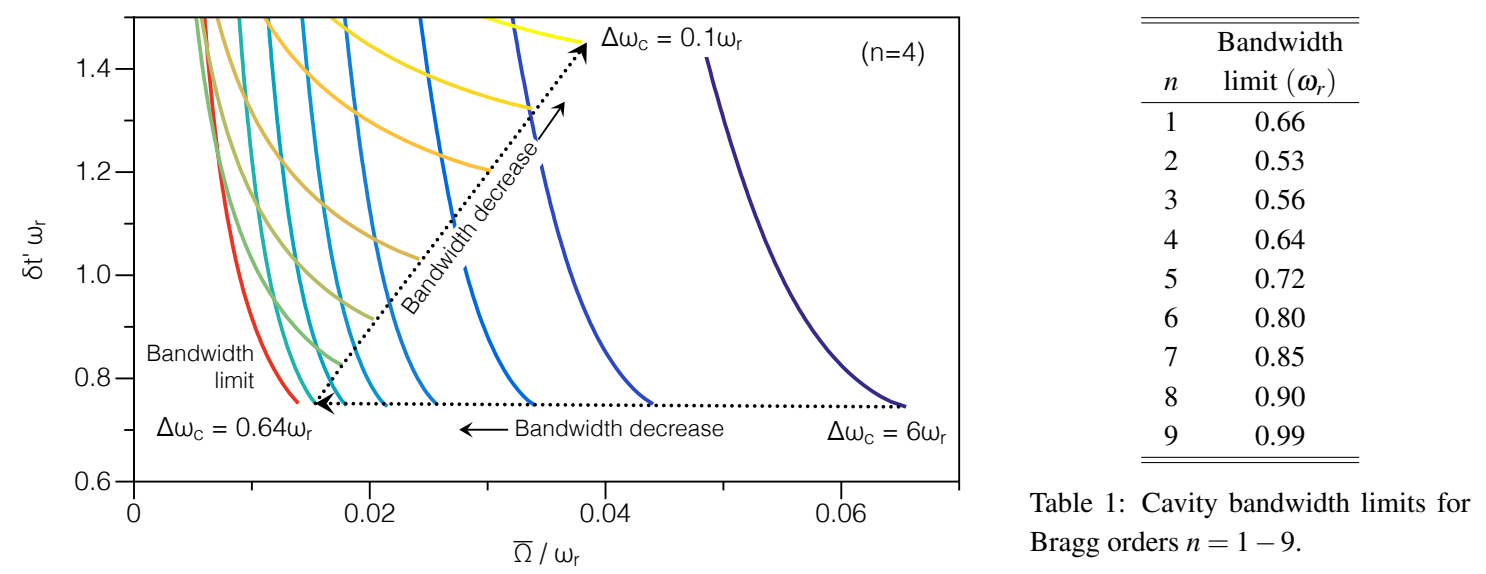

Figure 3: Fourth order $(n=4)$ Bragg diffraction $\pi$-pulses with losses below $5 \%$ in the $\delta t^{\prime}$ (interaction time) and $\bar{\Omega}$ (interaction strength) parameter space for cavities with different bandwidth (colour curves). The cavity operating at the bandwidth limit (red curve) offers maximum power enhancement of the interferometric beams and maintains the minimum interaction time unaffected.

\section{Cavity-assisted interferometer}

Having established a design limit on the cavity bandwidth based on optimising the beam splitter pulses, we now analyse the geometrical and optical properties of the full cavity-assisted interferometer. The biggest constraint on the cavity parameters is set by the fact that its eigenmode (the fundamental Hermite-Gauss mode, $\mathrm{HG}_{00}$ ) must accommodate the size of the atomic cloud, as it thermally expands during the measurement, whilst maintaining a geometrically stable configuration. Additionally, we will impose a requirement on the amount of suppression that the cavity provides of the first and second order spatial modes $\left(\mathrm{HG}_{01}\right.$ and $\left.\mathrm{HG}_{02}\right)$, in order to achieve a certain level of filtering of the interferometric beams. This analysis yields interferometer temperature limits as a function of three important design parameters.

The accumulated roundtrip Gouy phase shift in the cavity is $\zeta=2 \arccos \left(\sqrt{g_{1} g_{2}}\right)$, where $g_{i}=1-L / \mathscr{R}_{i}$ and $\mathscr{R}_{i}$ are the mirrors' radii of curvature. We will restrict our analysis to symmetric cavities, $\mathscr{R}_{1}=\mathscr{R}_{2} \Rightarrow g_{1}=g_{2}$, with the beam waist at the centre of the cavity. The total cavity $g$ factor $g_{c}=g_{1} g_{2}$ is an important parameter quantifying the cavity's geometrical stability. A strictly stable cavity verifies $0<g_{c}<1$, but in practice the cavity $g$-factor should not exceed a certain threshold $g_{\max }$ in order to maintain controllability [ [ ] ] . The roundtrip Gouy phase shift is an even more powerful parameter $(0<\zeta<2 \pi$ for a strictly stable cavity) because it is useful in quantifying if a particular set of higher order modes will be co-resonant with the fundamental mode. $\mathrm{The}_{\mathrm{H}} \mathrm{Gm}_{\mathrm{nm}}$ 
mode will accumulate a total Gouy phase shift of $(n+m) \zeta$ in a cavity roundtrip, leading to an optical suppression factor of that mode of [四:

$$
S_{n m}=\left(1+\left(\frac{2 \mathscr{F}}{\pi}\right)^{2} \sin ^{2}\left[(n+m) \frac{\zeta}{2}\right]\right)^{-\frac{1}{2}} .
$$

By setting a constraint of the type $S_{01,02} \leq S_{\max }$, we introduce a design requirement on the quality of the cavity as a spatial filter, as a low suppression factor of the first and second order modes means that the cavity will present a cleaner wavefront. The spatial filtering effect improves the quality of the beam inside the cavity regardless of the origin of the beam distortion, and applies in addition to other means of improving the input beam quality, such as pre-filtering, alignment, and mode matching.

Short cavities will be limited by the constraint set on the maximum $g$-factor, $g_{c} \leq g_{\max }$ (geometrical limit), as the smaller length comes at the price of putting the cavity very near the edge of geometrical instability for the required beam waist size. Larger cavities, on the other hand, while easily maintaining a stable configuration, will be limited by the constraints on cavity bandwidth and spatial filtering, $\Delta \omega_{c} \geq \Delta \omega_{\min }$ and $S_{01,02} \leq S_{\max }$ (optical limit), where $\Delta \omega_{\min }$ is the cavity bandwidth limit for the specific atomic transition, as listed in Table 1. Under these constraints, we can derive expressions for the maximal beam sizes allowed in the interferometer, distinguishing the two limits:

$$
\begin{aligned}
& w_{0, \text { geo }}(L)=\sqrt{\frac{L \lambda}{2 \pi} \cot \left(\frac{\arccos \sqrt{g_{\max }}}{2}\right)}, \\
& w_{0, \text { opt }}(L)=\left(\frac{\lambda^{2}}{4 \pi^{4} \Delta \omega_{\min }^{2}} \frac{2 c^{2} S_{\max }^{2}+\pi^{2} L^{2} \Delta \omega_{\min }^{2}\left(S_{\max }^{2}-1\right)+2 c S_{\max } \sqrt{c^{2} S_{\max }^{2}+\pi^{2} L^{2} \Delta \omega_{\min }^{2}\left(S_{\max }^{2}-1\right)}}{1-S_{\max }^{2}}\right)^{1 / 4}
\end{aligned}
$$

In the geometrical limit $w_{0, \text { geo }}<w_{0, \text { opt }}$, whereas in the optical limit $w_{0, \text { opt }}<w_{0, \text { geo }}$. Moreover, a length limit arises from the optical constraints:

$$
L_{\max }=\frac{\sqrt{3} c}{2 \pi \Delta \omega_{\min }} \frac{S_{\max }}{\sqrt{1-S_{\max }^{2}}} .
$$

The size of the atomic ensemble after a time $t$ during the experiment is characterised by a Gaussian distribution of width $\sigma_{t}=\left(\sigma_{0}^{2}+\sigma_{v}^{2} t^{2}\right)^{1 / 2}$, where $\sigma_{0}$ is the width of the initial position distribution and $\sigma_{v}=\left(k_{B} \mathscr{T}_{e} / M\right)^{1 / 2}$ is the width of the velocity distribution of temperature $\mathscr{T}_{e}$ and mass $M$, with $k_{B}$ the Boltzmann constant. Assuming that $\sigma_{v} t \gg \sigma_{0}$, we can approximate the size of the cloud at time $t$ by $\sigma_{t} \approx \sigma_{v} t$. The cloud size must be, at most, equal to the size of the cavity waist. Thus, the temperature of the atomic ensemble is limited by the maximum waist sizes in either the geometrical or the optical limits:

$$
\begin{aligned}
\mathscr{T}_{e, \mathrm{geo}} & \leq \frac{M \lambda L}{2 \pi k_{B} t^{2}} \cot \left(\frac{1}{2} \arccos \sqrt{g_{\max }}\right) \\
\mathscr{T}_{e, \mathrm{opt}} & \lesssim \frac{M c \lambda}{\pi^{2} k_{B} t^{2} \Delta \omega_{\min }} \frac{S_{\max }}{\sqrt{1-S_{\max }^{2}}}
\end{aligned}
$$


where the second equation is valid to first order in $L$. Note that we have assumed that the beam size to cloud size ratio is 1 . In the future we aim to fully model the effect that the imperfect optical wavefronts - spatially filtered by the cavity - have on the atomic transitions, therefore quantifying the effect that the optical suppression ratio of higher order modes and the beam size to cloud size ratio have on the beam splitters.

\section{Conclusions}

We have studied, by means of a numerical model, the Bragg scattering of atoms from the time-distorted pulses resonating in an optical cavity. We have found a lower bound in the cavity bandwidth which maximises power enhancement of the beam splitter pulses and keeps the minimum interaction time unaffected. By introducing constraints on cavity stability and spatial filtering, through the total cavity $g$-factor and the optical suppression factor of the first and second higher order modes respectively, we have derived interferometer temperature limits as a function of cavity length, as well as a cavity length limit. These limits highlight the incompatibility between achieving both good spatial filtering and large beam sizes. Long baseline experiments employing large cavities to assist the beam splitter pulses, such as MIGA, will need to operate with low finesses in order to compensate for their naturally lower bandwidth, and will likely offer both limited power enhancement and poor spatial filtering due to this. We found that $L_{\max }$ scales inversely with the cavity bandwidth and, to first order, directly with the suppression factor of higher order modes. Additionally, we found that the minimum cavity bandwidth increases linearly with the order of the diffraction process for $n \geq 2$. Hence, large momentum transfer beam splitters will be more prohibitive for long baseline experiments, with the upper bound on cloud temperature scaling with $1 / n$. In such experiments, the cavity finesse will greatly affect the minimum $\pi$-pulse duration and power requirements, as well as the level of spatial filtering achievable by the cavity.

\section{References}

[1] B. Canuel, A. Bertoldi, L. Amand, E. B. di Pozzo, B. Fang, R. Geiger, J. Gillot, S. Henry, J. Hinderer, D. Holleville, G. Lefèvre, M. Merzougui, N. Mielec, T. Monfret, S. Pelisson, M. Prevedelli, S. Reynaud, I. Riou, Y. Rogister, S. Rosat, E. Cormier, A. Landragin, W. Chaibi, S. Gaffet, and P. Bouyer, Exploring gravity with the MIGA large scale atom interferometer, arXiv: 703.02490, (2017).

[2] The LIGO Scientific Collaboration, Advanced LIGO, Class. Quantum Grav. 32, 074001 (2015) [arXiv:14[11.4547].

[3] M. Dovale-Álvarez, D. D. Brown, A. W. Jones, C. M. Mow-Lowry, H. Miao, and A. Freise, Fundamental limitations of cavity-assisted atom interferometry, Phys. Rev. A 96, 053820 (2017) [arXiv: 710.02448 ]

[4] P. Meystre, Atom Optics (Springer Series on Atomic, Optical, and Plasma Physics), Springer (2001).

[5] H. Müller, S.-W. Chiow, and S. Chu, Atom-wave diffraction between the Raman-Nath and the Bragg regime: Effective Rabi frequency, losses, and phase shifts, Phys. Rev. A 77, 023609 (2008) [arXiv:07042627].

[6] C. Keller, J. Schmiedmayer, A. Zeilinger, T. Nonn, S. Dürr, and G. Rempe, Adiabatic following in standing-wave diffraction of atoms, Appl. Phys. B 69, 303 (1999). 
[7] A. Araya, K. Tsubono, K. Suehiro, M.-K. Fujimoto, M. Ohashi, N. Mio, and S. Telada, Optical mode cleaner with suspended mirrors, Applied Optics 36, 1446 (1997).

[8] H. Wang, M. Dovale-Álvarez, C. Collins, D. D. Brown, M. Wang, C. M. Mow-Lowry, Sen Han, and A. Freise, Feasibility of near-unstable cavities for future gravitational wave detectors, Phys. Rev. D 97, 022001 (2018) [arXiv: 17 1.05177]]. 